\title{
The effects of phosphanegold(I) thiolates on the biological properties of Acanthamoeba castellanii belonging to the T4 genotype
}

\author{
Ruqaiyyah Siddiqui ${ }^{1}$, Farhat Abjani ${ }^{1}$, Chien Ing Yeo², Edward R. T. Tiekink ${ }^{2}$ and Naveed Ahmed Khan ${ }^{1 *}$
}

\begin{abstract}
Background: Gold compounds have shown promise in the treatment of non-communicable diseases such as rheumatoid arthritis and cancer, and are considered of value as anti-microbial agents against Gram-negative and Gram-positive bacteria, and have anti-parasitic properties against Schistosoma mansoni, Trypanosoma brucei, Plasmodium falciparum, Leishmania infantinum, Giardia lamblia, and Entamoeba histolytica. They are known to affect enzymatic activities that are required for the cellular respiration processes.

Methods: Anti-amoebic effects of phosphanegold(I) thiolates were tested against clinical isolate of $A$. castellanii belonging to the T4 genotype by employing viability assays, growth inhibition assays, encystation assays, excystation assays, and zymographic assays.

Results: The treatment of A. castellanii with the phosphanegold(I) thiolates tested (i) had no effect on the viability of $A$. castellanii as determined by Trypan blue exclusion test, (ii) did not affect amoebae growth using PYG growth medium, (iii) did not inhibit cellular differentiation, and (iv) had no effect on the extracellular proteolytic activities of A. castellanii.

Conclusion: Being free-living amoeba, A. castellanii is a versatile respirator and possesses respiratory mechanisms that adapt to various aerobic and anaerobic environments to avoid toxic threats and adverse conditions. For the first time, our findings showed that $A$. castellanii exhibits resistance to the toxic effects of gold compounds and could prove to be an attractive model to study mechanisms of metal resistance in eukaryotic cells.
\end{abstract}

Keywords: Acanthamoeba, Gold compounds, Cytotoxicity assays, Zymography, Encystation, Excystation

\section{Background}

Acanthamoeba is a free living pathogenic protist that can cause cutaneous lesions, a vision-threatening keratitis, and a rare but fatal infection of the brain, identified as granulomatous amoebic encephalitis [1-4]. Acanthamoeba keratitis infection is of explicit concern given the rise in the number of wearers of contact lenses worldwide, a population susceptible to this infection. Treatment involves hourly topical application of a mixture of drugs comprising of polyhexamethylene biguanide or chlorhexidine digluconate together with propamidine isethionate or hexamidine. Moreover,

\footnotetext{
* Correspondence: naveed5438@gmail.com

${ }^{1}$ Department of Biological Sciences, Faculty of Science and Technology,

Sunway University, 47500 Bandar Sunway, Selangor, Malaysia

Full list of author information is available at the end of the article
}

chloramphenicol or neomycin is also given to prevent mixed bacterial infection [5]. Treatment lasts for several months $[5,6]$. Furthermore, the treatment is problematic and cumbersome, in part due to the ability of this facultative parasite to go through phenotypic interchanging into a double-walled cyst form, which is impervious to many anti-microbial drugs and harsh conditions, and an active vegetative trophozoite stage that is more vulnerable to anti-microbials, often leading to recurrence of infection [7-9]. Consequently, there is a crucial need to develop anti-microbials targeting both the cyst stage and the trophozoite stage of Acanthamoeba.

Gold compounds have been well recognised for their putative properties and potential medical applications $[10,11]$. For example, the assessment of the potential anti-cancer activity and the determination of signalling 
pathways for apoptosis of phosphane gold(I) carbonimidothioates, $\mathrm{Ph}_{3} \mathrm{PAu}[\mathrm{SC}(\mathrm{OR})=\mathrm{NPh}], \mathrm{R}=\mathrm{Me}, \mathrm{Et}$ and iPr, and related species have been carried out recently [12-14], see Fig. 1 for chemical structures. Moreover, closely related compounds have shown potential as anti-microbial agents against Gram-positive bacteria [15]. Gold(I) compounds have potential medical applications and shown to possess anti-tumour activities $[16,17]$, anti-parasitic [18] and anti-microbial activities [19-21] via a variety of mechanisms including respiration. In this study, for the first time, we determined the effects of phosphanegold(I) thiolates, AAu1-AAu3, Fig. 1, on a keratitis-causing isolate of $A$. castellanii belonging to the T4 genotype. Furthermore, the effects on viability, growth, encystation and excystation are examined.

\section{Methods}

\section{Chemicals}

All chemicals were purchased from Sigma Labs (Poole, Dorset, England), unless otherwise stated. The phosphanegold(I) thiolates, AAu1-AAu3, were prepared and characterised using methodology as previously described [14]. The molecular structures and weights of AAu1AAu3 are given in Fig. 1. A stock solution $(10 \mathrm{mM})$ was prepared and stored at $-20{ }^{\circ} \mathrm{C}$ until used. Control cultures contained the same volume of respective solvents.

\section{Cultures of $\mathrm{A}$. castellanii}

A. castellanii belonging to the T4 genotype (ATCC 50492) is a clinical isolate that was initially isolated from a keratitis patient and grown in $75 \mathrm{~cm}^{2}$ tissue culture flasks in $10 \mathrm{~mL}$ at a cell density of $5 \times 10^{5}$ cells per $\mathrm{mL}$ in PYG medium [proteose peptone $0.75 \%(\mathrm{w} / \mathrm{v})$, yeast extract $0.75 \%(\mathrm{w} / \mathrm{v})$ and glucose $1.5 \%(\mathrm{w} / \mathrm{v})]$ without

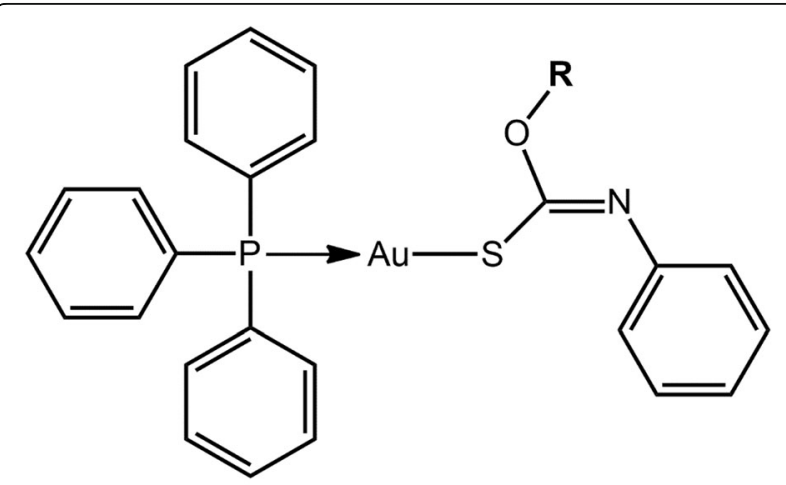

AAu1: $\mathbf{R}=$ Me; M. wt: 625.45

AAu2: $\mathbf{R}=\mathrm{Et} ; \quad$ M. wt: 639.48

AAu3: $\mathbf{R}=\mathrm{iPr} ; \quad$ M. wt: 653.51

Fig. 1 Chemical diagrams, abbreviations and molecular weights for AAu1-AAu3 shaking at $30{ }^{\circ} \mathrm{C}$ as described previously $[22,23]$. At this cell density, parasites reach confluency within $48 \mathrm{~h}$. Active trophozoites are attached to the bottom of the flasks while any dormant cells are non-adherent in the supernatant. To obtain trophozoites, supernatant was aspirated and $10 \mathrm{~mL}$ of RPMI-1640 was added. Next, flasks were placed on ice for $20 \mathrm{~min}$ to detach bound amoebae followed by gentle tapping and observed under the inverted microscope to ensure amoebae detachment had occurred. Finally, the parasites were collected in $50 \mathrm{~mL}$ tubes, followed by centrifugation at $1500 \times g$ for $5 \mathrm{~min}$, resuspended in one $\mathrm{mL}$ of RPMI-1640 and used in experiments.

\section{Amoebicidal assays}

To determine amoebicidal activity of AAu1-AAu3, A. castellanii trophozoites $\left(5 \times 10^{5}\right.$ amoebae $/ 0.5 \mathrm{~mL} /$ well $)$ were incubated in RPMI-1640 with various concentrations of AAu1-AAu3 in 24-well plates as described previously [20-24]. Plates were incubated at $37^{\circ} \mathrm{C}$ for $24 \mathrm{~h}$. Following this incubation, amoebae viability was determined by adding $0.1 \%$ Trypan blue and number of live (non-stained) and dead (stained) A. castellanii were enumerated using a haemocytometer. The counts from $A$. castellanii incubated with RPMI-1640 alone, and the solvent alone (chloroform) were used as controls. Data are represented as the mean \pm standard error of at least three independent experiments. To determine whether the effects of AAu1-AAu3 are irreversible, A. castellanii, $5 \times 10^{5}$ trophozoites, were incubated with AAu1-AAu3 for $24 \mathrm{~h}$ as described above. After this incubation, amoebae were centrifuged for $10 \mathrm{~min}$ at $1,000 \mathrm{xg}$ and supernatant was aspirated, followed by the addition of $0.5 \mathrm{~mL}$ of RPMI-1640. This process was repeated 3X to remove extracellular AAu1-AAu3. Finally, A. castellanii were re-suspended in PYG as a food source and inoculated in 24-well plates. Plates were incubated at $37{ }^{\circ} \mathrm{C}$ for up to $72 \mathrm{~h}$ and re-emergence of trophozoites was considered as viable amoebae, and absence of trophozoites was considered as non-viable amoebae. In some experiments, plates were incubated for up to a week to observe the emergence of viable trophozoites.

\section{Amoebistatic assays}

To determine the effects of AAu1-AAu3 on the growth of $A$. castellanii, assays were performed by exposing $5 \times$ $10^{5}$ trophozoites to different concentrations of AAu1AAu3 in growth medium, i.e., PYG in 24-well plates. Next, the plates were incubated at $30{ }^{\circ} \mathrm{C}$ for $48 \mathrm{~h}$. For controls, $5 \times 10^{5}$ trophozoites were inoculated in 100\% PYG medium, 100\% non-nutritive PBS and respective amounts of solvents plus PYG medium and incubated in the above-mentioned conditions. After this incubation, the number of amoebae was 
determined by haemocytometer counting. All experiments were performed at least three times in duplicate.

\section{Preparation of A. castellanii cysts and excystation assays}

To prepare A. castellanii cysts, encystation was induced by inoculating $5 \times 10^{6} \mathrm{~A}$. castellanii trophozoites onto non-nutrient agar plates [prepared using 3\% (w/v) bacteriological agar] and incubating at $30{ }^{\circ} \mathrm{C}$ for up to 14 days [25]. Food deprivation resulted in trophozoite transformation into the cyst form. Next, $10 \mathrm{~mL}$ of PBS was added to each plate. Cysts were then gently scraped off the agar surface using a cell scraper. PBS containing cysts was collected in $15 \mathrm{~mL}$ tube and centrifuged at $3000 \times g$ for $10 \mathrm{~min}$ to pellet cysts. The supernatant was aspirated and cysts resuspended in RPMI-1640, enumerated using a haemocytometer and used in experiments. To determine the effects of AAu1-AAu3 on excystation, assays were performed by inoculating $A$. castellanii cysts $\left(5 \times 10^{4}\right.$ cysts per $\mathrm{mL}$ PYG per well of 24-well plates) in the presence or absence of different concentrations of AAu1-AAu3. Plates were incubated at $30{ }^{\circ} \mathrm{C}$ and observed every $24 \mathrm{~h}$ under the inverted microscope for the emergence of viable trophozoites for up to $72 \mathrm{~h}$.

\section{Encystation assays}

Encystation assays were performed as described previously [25]. Briefly, $2 \times 10^{6}$ amoebae were incubated in $0.5 \mathrm{~mL}$ of PBS containing $50 \mathrm{mM} \mathrm{MgCl}_{2}$ and $10 \%$ glucose (i.e., encystation trigger) per well of 24-well plates. The plates were incubated at $30{ }^{\circ} \mathrm{C}$ for $72 \mathrm{~h}$ without shaking. After this incubation, amoebae viability was quantified using a haemocytometer via Trypan blue exclusion assay. Next, SDS (0.5\% final conc.) was added for $10 \mathrm{~min}$. At this concentration, SDS solubilizes amoebae trophozoites but not cysts. Finally cysts were enumerated using a haemocytometer and used in experiments. To determine the effects of AAu1-AAu3 on encystation, assays were performed in the presence of different concentrations of drugs. Briefly $2 \times 10^{6}$ amoebae were incubated in PBS with various concentrations of drugs and incubated at room temperature for $20 \mathrm{~min}$. Following this, $50 \mathrm{mM}$ $\mathrm{MgCl}_{2}$ and $10 \%$ glucose was added as a trigger for encystation and plates were incubated at $30^{\circ} \mathrm{C}$ for $72 \mathrm{~h}$. Finally, parasites counts were determined using a haemocytometer. Amoebae incubated without inhibitors and encystation trigger were used as controls. The respective amounts of solvents were used as solvent controls.

\section{Zymographic assays}

The extracellular proteolytic activities of Acanthamoeba were determined using zymographic assays as previously described [26]. Briefly, A. castellanii were incubated in the presence or absence of various concentrations of AAu1AAu3 for $24 \mathrm{~h}$. Next day, cell-free supernatants (CM, conditioned medium) were collected by centrifugation. The CM were electrophoresed on sodium dodecyl sulfatepolyacrylamide gel electrophoresis (SDS-PAGE) containing gelatin $(2 \mathrm{mg} / \mathrm{mL})$ as a protease substrate as previously described [26]. Following electrophoresis, gels were washed in $2.5 \%$ Triton $\mathrm{X}-100(\mathrm{w} / \mathrm{v})$ for $60 \mathrm{~min}$, then incubated in developing buffer $(50 \mathrm{mM}$ Tris- $\mathrm{HCl}, \mathrm{pH} 7.5$, containing $10 \mathrm{mM} \mathrm{CaCl}{ }_{2}$ ) at $37{ }^{\circ} \mathrm{C}$ overnight. Next day, gels were stained with Coomassie Brilliant Blue. Areas of gelatin digestion were visualised as non-staining regions in the gel.

\section{Statistical analysis}

Statistical significance for differences was evaluated using 2 sample $t$-test; two-tailed distribution, comparing the mean of two independent groups in Excel. A critical value of $\mathrm{P}<0.05$ was used for all analysis. For graphical representation of the data, $y$-axis error basis indicate the standard error of the data for each point on the figure.

\section{Results}

Phosphanegold(I) thiolates, AAu1-AAu3, did not affect A. castellanii trophozoites viability

To ascertain the effects of AAu1-AAu3, amoebicidal assays were performed as stated in Materials and Methods. The results revealed that AAu1-AAu3 did not exhibit anti-amoebic effects against $A$. castellanii trophozoites (Fig. 2a and b). In the presence of 100, 200 and $300 \mu \mathrm{M}$ AAul, the number of viable amoebae was $3.41 \times 10^{5} \pm$ $1.12 \times 10^{4}, 2.84 \times 10^{5} \pm 5.51 \times 10^{3}$ and $2.62 \times 10^{5} \pm 3.47 \times$ $10^{4}$, respectively. However, this was not significant when compared to the respective solvent controls $(5,10$ and $15 \mu \mathrm{L}$ chloroform). Likewise, for 100,200 and $300 \mu \mathrm{M}$ $\mathrm{AAu} 2$, the number of viable amoebae was $2.88 \times 10^{5} \pm$ $1.75 \times 10^{4}, 2.72 \times 10^{5} \pm 4.73 \times 10^{4}$ and $2.30 \times 10^{5} \pm 2.14 \times$ $10^{4}$, respectively. For 100,200 and $300 \mu \mathrm{M}$ AAu3, the number of viable amoebae was $2.94 \times 10^{5} \pm 1.56 \times 10^{4}$, $2.76 \times 10^{5} \pm 3.09 \times 10^{4}$ and $2.23 \times 10^{5} \pm 3.39 \times 10^{4}$, respectively (Fig. 2a). Overall, the results showed no effects of AAu1-AAu3 on amoebae viability.

\section{Phosphanegold(I) thiolates, AAu1-AAu3, did not exhibit amoebistatic effects against $A$. castellanii trophozoites} Amoebistatic assays were performed in the presence or absence of AAu1-AAu3. When incubated in 100\% growth medium, the number of amoebae increased from $5 \times 10^{5}$ to $8.78 \times 10^{5} \pm 3.21 \times 10^{4}$ (Fig. 3). In contrast, amoebae incubated in non-nutritive RPMI medium had no growth stimulatory effect but exhibited reduced number of amoebae i.e., the amoebae count decreased from $5 \times 10^{5}$ to $3.29 \times 10^{5} \pm 6.63 \times 10^{4}$ (Fig. 3). For AAu1-AAu3, the results revealed that there were no amoebistatic effects against $A$. castellanii even at $300 \mu \mathrm{M}$ concentrations. For $\mathrm{AAu} 1-\mathrm{AAu} 3$, the number of amoebae increased from $5 \times$ 


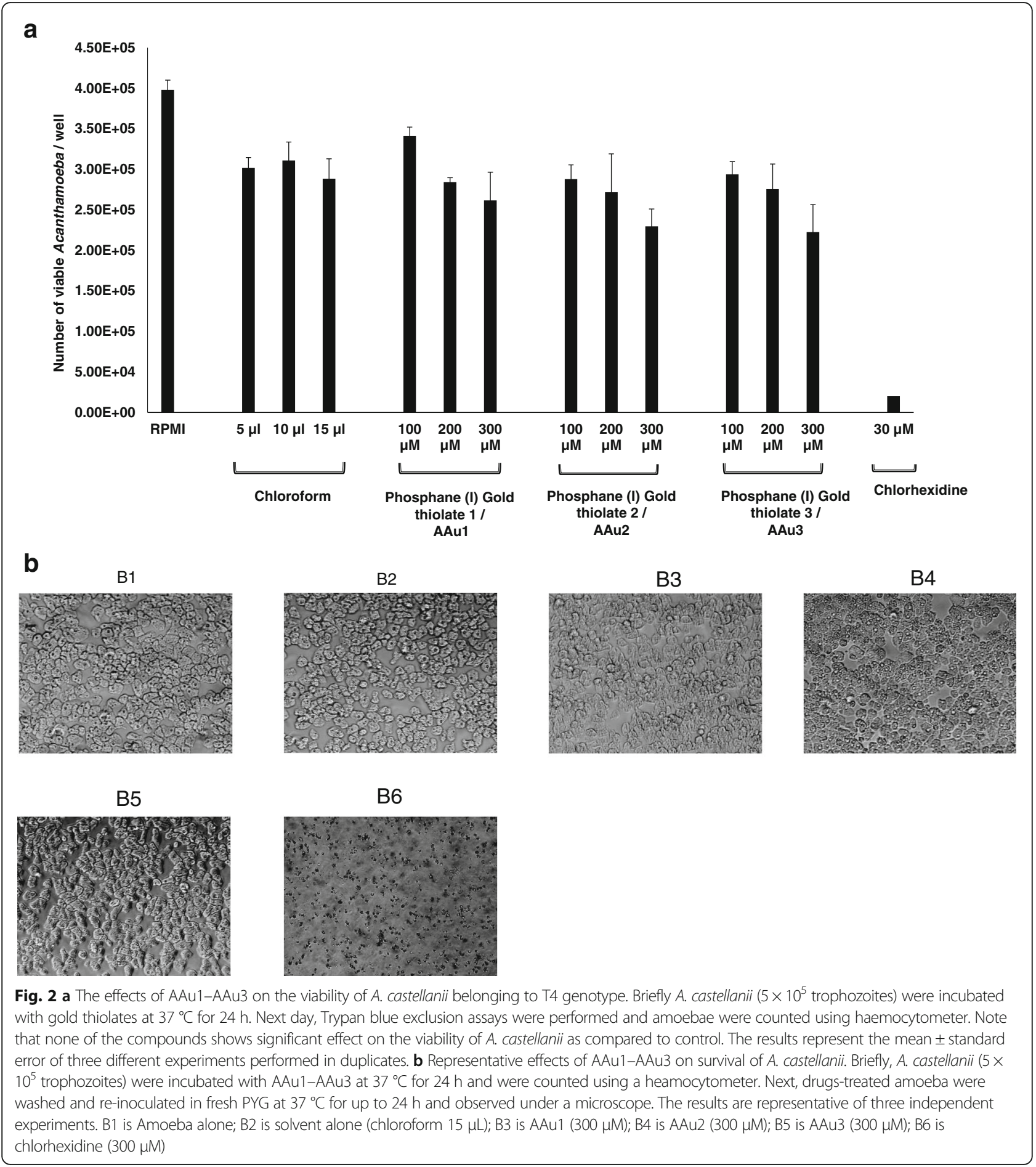

$10^{5}$ to $9.56 \times 10^{5} \pm 8.42 \times 10^{4}, 7.02 \times 10^{5} \pm 9.38 \times 10^{4}$ and $9.85 \times 10^{5} \pm 3.07 \times 10^{4}$, respectively at $300 \mu \mathrm{M}$.

\section{Phosphanegold(I) thiolates, AAu1-AAu3, did not affect} excystation in A. castellanii

When incubated in growth medium, the number of amoebae increased from $5 \times 10^{4}$ to $3.91 \times 10^{5} \pm 1.63 \times$
$10^{4}$ as compared to $5 \times 10^{4}$ to $1.24 \times 10^{5} \pm 1.38 \times 10^{4}$ in RPMI medium, which is a non-nutritive medium (Fig. 4a). However, for AAu1-AAu3, the number of amoebae increased from $5 \times 10^{4}$ to $3.50 \times 10^{5} \pm 1.63 \times$ $10^{4}, \quad 3.73 \times 10^{5} \pm 2.50 \times 10^{4}$ and $3.21 \times 10^{5} \pm 2.81 \times 10^{4}$, respectively at $300 \mu \mathrm{M}$ (Fig. 4a). Nonetheless, this was not significant when compared to the respective growth 


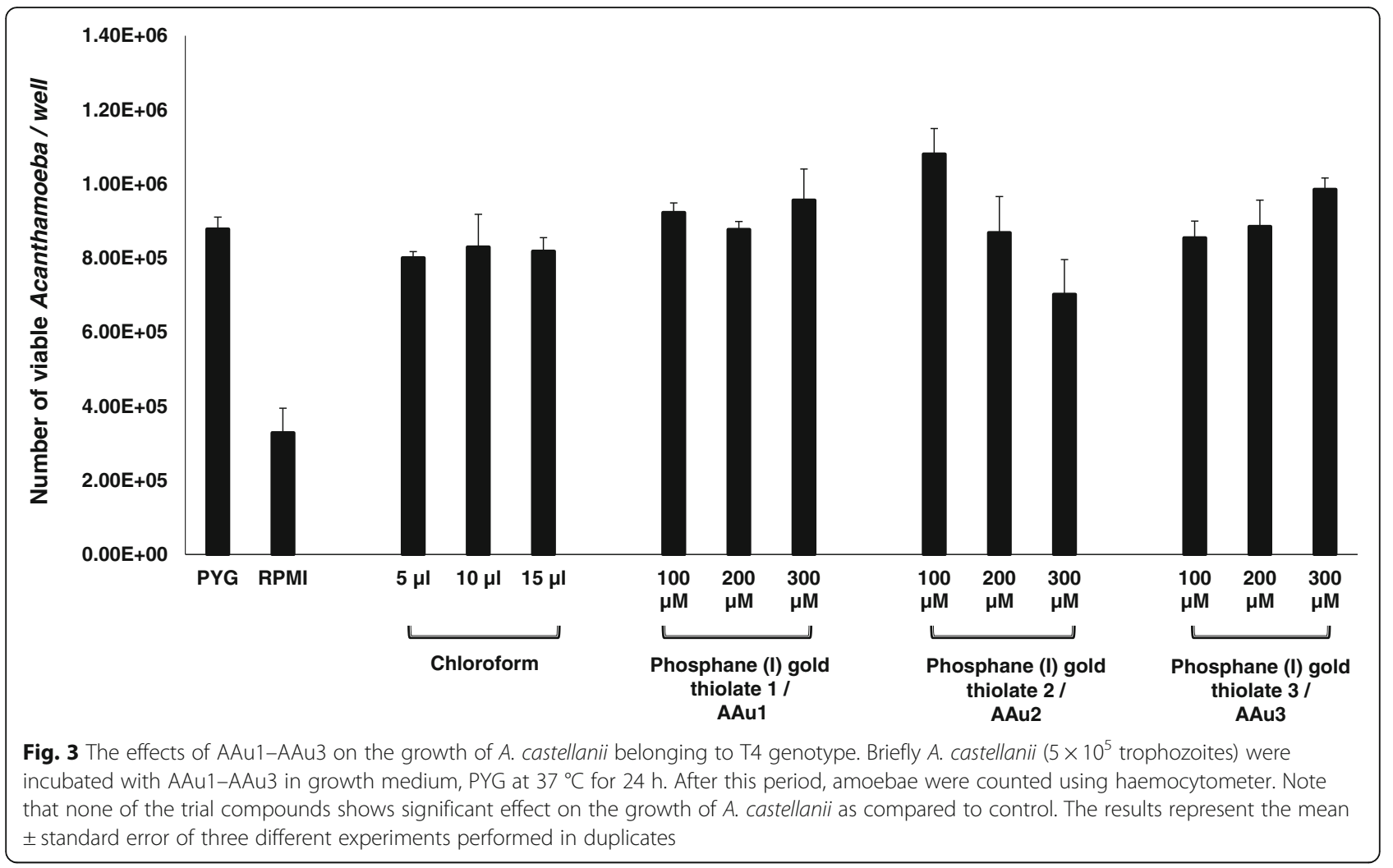

medium control and the results revealed that none of the compounds tested had any effects on excystation, and amoebae were able to excyst at rates comparable to controls (Fig. 4b).

\section{Phosphanegold(I) thiolates, AAu1-AAu3, did not affect encystation in A. castellanii}

To determine the effects of AAu1-AAu3 on A. castellanii encystation, assays were performed in the presence and absence of these compounds. When incubated in encystation medium, the number of amoebae decreased from $5 \times 10^{5}$ to $1.73 \times 10^{5} \pm 2.50 \times 10^{3}$ (Fig. 5). However, for AAu1-AAu3, the number of amoebae was reduced from $5 \times 10^{5}$ to $1.18 \times 10^{5} \pm 4.75 \times 10^{4}, \quad 1.17 \times 10^{5} \pm$ $2.06 \times 10^{4}$ and $1.17 \times 10^{5} \pm 1.44 \times 10^{4}$, respectively, at $300 \mu \mathrm{M}$ (Fig. 5). However, this was not significant when compared to the respective encystation medium control. The results revealed that none of the trial compounds tested had any effects on encystation.

Phosphanegold(I) thiolates, AAu1-AAu3, did not effect A. castellanii extracellular proteolytic activity

To determine the effect of AAu1-AAu3 on the extracellular proteases of $A$. castellanii, zymographic assays were performed using gelatin as substrate as described in materials and methods. In the absence of any trial compound, A. castellanii exhibited proteolytic activities and a visible band of $140 \mathrm{kDa}$ was observed (Fig. 6). Similarly, both, A. castellanii treated in the presence of different concentrations of AAu1-AAu3 and in RPMI alone exhibited extracellular proteases at similar levels (Fig. 6).

\section{Discussion}

Gold(I) complexes have potential medical applications $[10,11]$. Thus, gold(I) derivatives have been explored for anti-tumour activity [16, 17] as well as anti-parasitic [18] and anti-microbial agents [19-21]. Gold has properties such as high thermal/chemical stability and resistant to oxidation, yet is mechanically soft with high electric conductivity enabling its applications in several disciplines ranging from healthcare to engineering. For example, gold compounds have been successfully used in the treatment of rheumatoid arthritis and are shown to slow down the progression of rheumatic disorder [27, 28]. Many of the biologically active gold(I) compounds contain thiolates and/or phosphane as ligands [10, 11, 16, $17,21]$ and inhibit thioredoxin reductase [29, 30]. More recently, it is shown that the gold(I) compounds exhibit anti-parasitic activities such as targeting Schistosoma mansoni [31], Trypanosoma brucei [32], Echinococcus granulosus [33], Plasmodium falciparum [34], Leishmania infantinum [35] Giardia lamblia [36], and Entamoeba histolytica [37]. Furthermore, it was shown that 


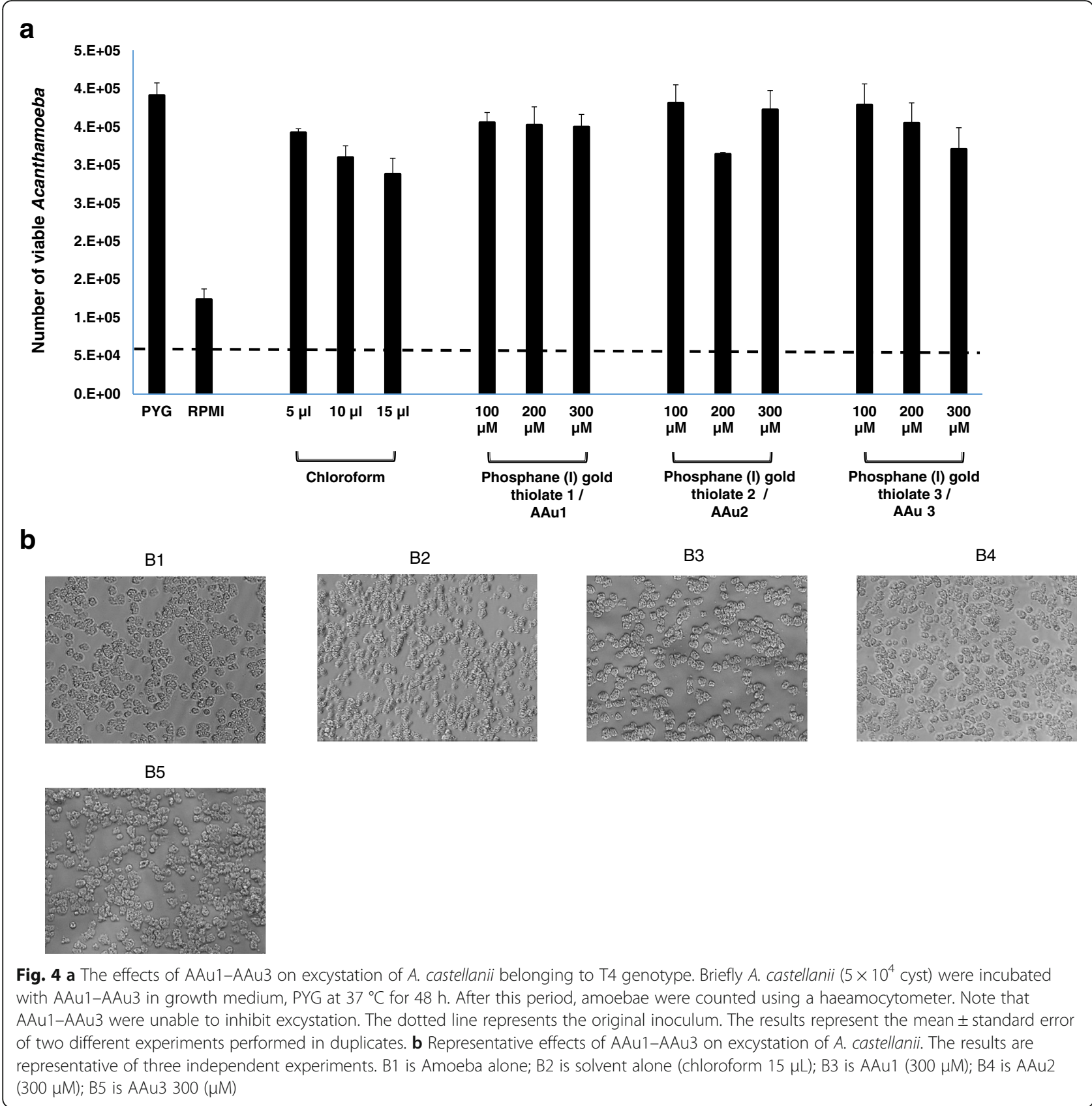

gold(I) compounds target E. histolytica by inhibiting thioredoxin reductase activity [37]. The anti-bacterial activities of gold(I) compounds showed that these compounds affect Clostridium difficile and Treponema denticola by disrupting the selenium metabolism by targeting selenoproteins required for energy [38, 39], while Staphylococcus aureus growth is inhibited by gold(I) compounds [40]. Other studies proposed targets including the inhibition of mitochondrial enzymes and of the proteasome compounds [41, 42] and the inhibition of the zinc finger protein poly (adenosine diphosphate (ADP) ribose) polymerase 1 (PARP-1) [43, 44]. Notably,
PARP's are crucial proteins that are important in drug resistance in cancer as they play an essential role in DNA repair by detecting DNA strand breaks and catalyzing poly (ADP-ribosylation) [45]. Other biological targets of gold(I) compounds with prokaryotic and eukaryotic cells are yet to be discovered.

Based on these findings, it was logical to test the antiamoebic effects of phosphanegold(I) thiolates, AAu1AAu3, on the biological properties of A. castellanii belonging to the $\mathrm{T} 4$ genotype. The results revealed that AAu1-AAu3 did not show any effects on the biological properties of the parasite. This was determined by 


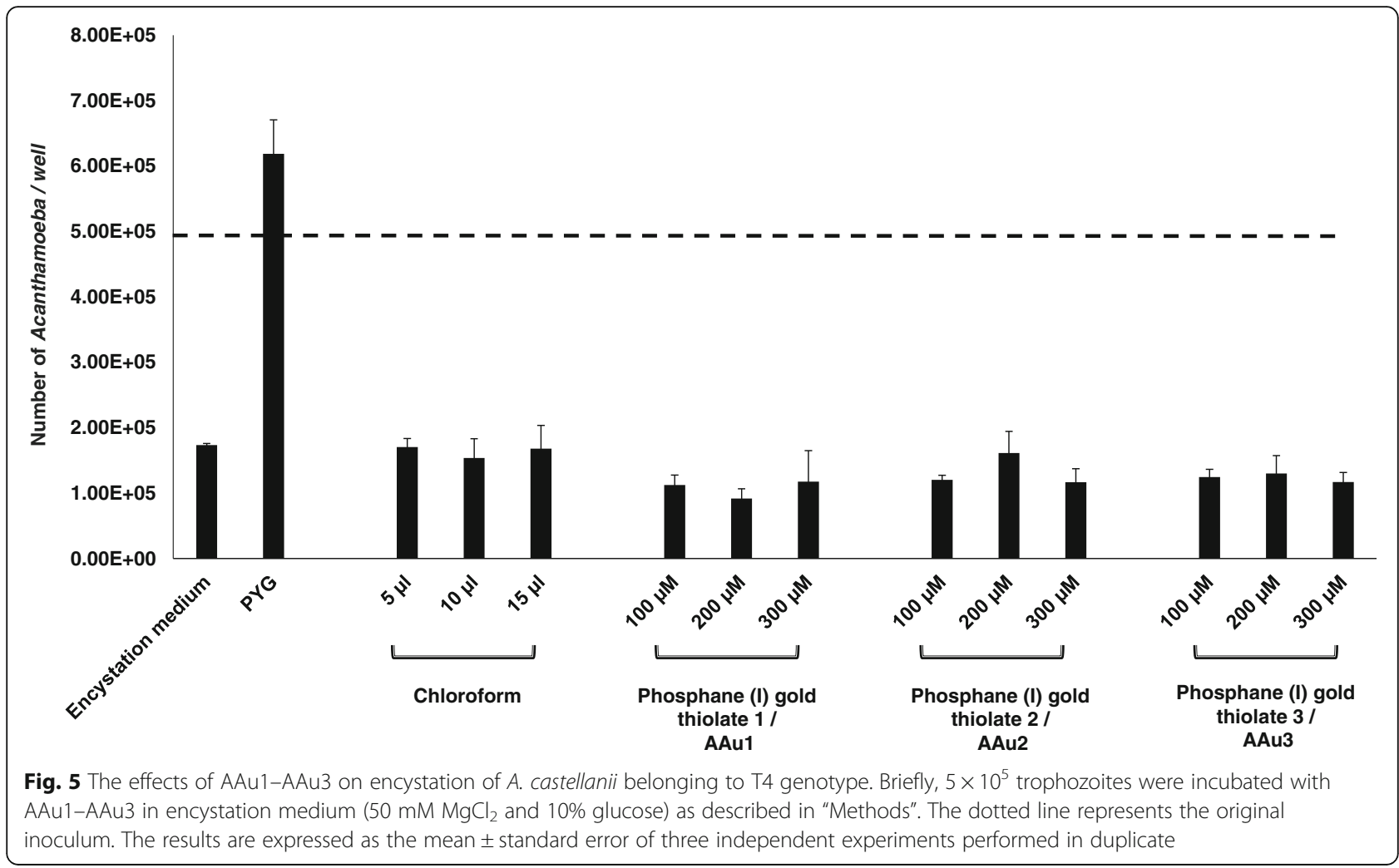

performing (i) viability assays using Trypan blue exclusion test, (ii) amoebae growth using PYG growth medium, (iii) cellular differentiation using encystation and excystation assays and (iv) enzymatic activities by determining extracellular proteases profiles. The reported results are highly reproducible and consistently showed that AAu1-AAu3 do not affect the biological properties of $A$. castellanii. There could be several explanations for the findings observed in this study. For example, the mode of action of gold requires it to enter the cell, via the hydrophobic cell membrane, to produce damage, most likely through transmembrane proteins that may be different in A. castellanii. Notably, gold(I) compounds are well known to affect enzymatic activities that are required for the cellular respiration processes. Being one of the most ubiquitous protists, the natural

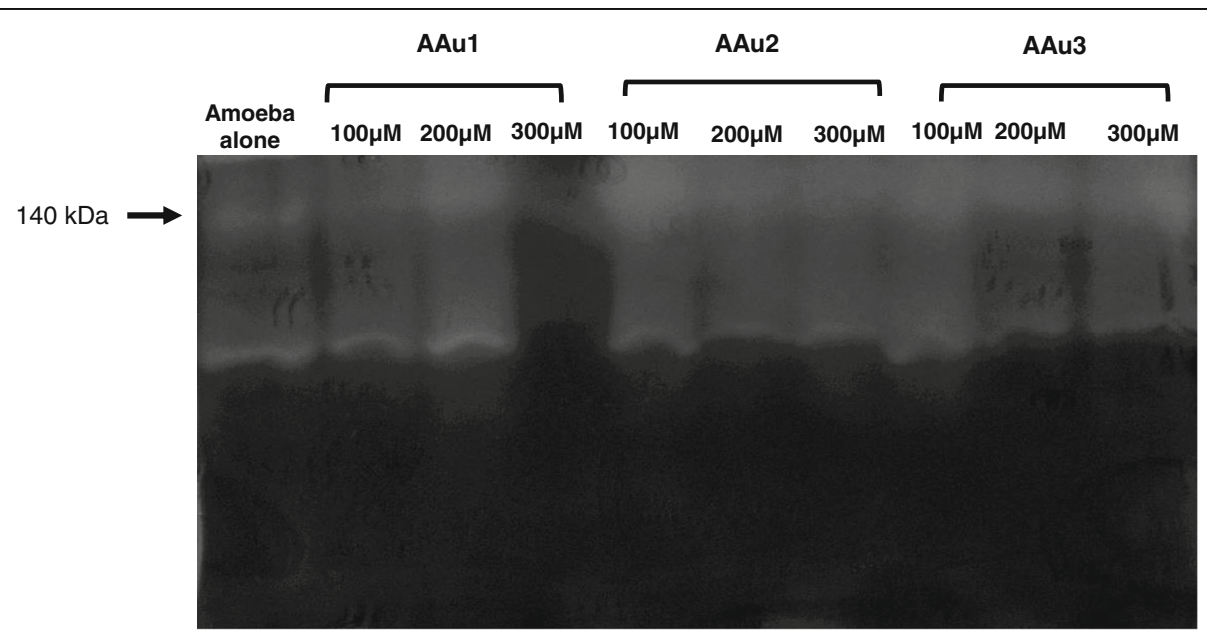

Fig. 6 The effects of AAu1-AAu3 on extracellular proteolytic activity of A. castellanii belonging to T4 genotype. Zymographic assays were performed using gelatin as a substrate to determine the effects of AAu1-AAu3 on extracellular proteases of A. castellanii using 100, 200 and $300 \mu \mathrm{M}$ concentrations. The results revealed that none of AAu1-AAu3 inhibited A. castellanii proteases when compared with amoeba in RPMI alone. The results are representative of three independent experiments 
habitat of Acanthamoeba is the environment with diverse respiratory mechanisms and wide exposure to metals, thus Acanthamoeba is likely to possesses mechanisms to inhibit the toxic effects exerted by metals. $A$. castellanii is well known as a versatile respirator and possesses several mitochondria per cell and respiratory mechanisms that adapt to various aerobic and anaerobic environments to dodge toxic threat and adverse conditions. It is possible that the toxic effects of metals are compensated by switching the type of respiration or the use of an efflux system to rid toxic metals. Future studies are needed to test higher concentration of phosphanegold(I) thiolates compounds and/or in combining phosphanegold(I) thiolates with current anti-amoeba drugs, such as chlorhexidine to determine their improved efficacy against pathogenic Acanthamoeba. Overall, these findings suggest that Acanthamoeba exhibits resistance to toxic effects of gold(I) compounds and could prove to be an attractive model to study mechanisms of metal resistance in eukaryotic cells.

\section{Conclusions}

Although gold compounds have shown promise in the treatment of non-communicable diseases such as rheumatoid arthritis, anti-tumour activities, as well as antibacterial properties, and anti-parasitic properties against protozoan pathogens, $T$. brucei, $P$. falciparum, $L$. infantinum, G. lamblia, and E. histolytica, often by targeting respiration pathways, our studies demonstrated that $A$. castellanii exhibited resistance against their toxic effects. The gold derivatives tested had no effect on the viability of $A$. castellanii, did not inhibit amoebae growth, or cellular differentiation processes or extracellular proteolytic activities. As Acanthamoeba is a versatile respirator, it can adapt to various aerobic and anaerobic environments to avoid toxic threats. Our studies suggest that Acanthamoeba could prove to be a useful model to study mechanisms of metal resistance in eukaryotic cells.

\section{Acknowledgements}

Not applicable.

\section{Funding}

This work was supported by the University Research Grant Scheme No. 201503, Sunway University, Malaysia.

\section{Availability of data and materials}

For data requests, please contact Distinguished Professor Naveed Ahmed Khan (Naveed5438@gmail.com).

\section{Authors' contributions}

RS conceived the study. CIY and ERTT synthesised and characterised AAu1AAu3. FA and NAK carried out all biological experiments. FA and RS collected relevant literature and wrote the first draft. NAK corrected the original manuscript. All authors approved the final manuscript.

\section{Competing interests}

The authors declare that they have no competing interests.
Consent for publication

Not applicable.

Ethics approval and consent to participate

Not applicable.

\section{Author details}

'Department of Biological Sciences, Faculty of Science and Technology, Sunway University, 47500 Bandar Sunway, Selangor, Malaysia. ${ }^{2}$ Research Centre for Crystalline Materials, Sunway University, 47500 Bandar Sunway, Selangor, Malaysia.

Received: 17 November 2016 Accepted: 9 February 2017

Published online: 03 April 2017

References

1. Khan NA. Pathogenesis of Acanthamoeba infections. Microb Pathogen. 2003; 34:277-85.

2. Khan NA. Acanthamoeba: biology and increasing importance in human health. FEMS Microbiol Rev. 2006:30:564-95.

3. Visvesvara GS, Moura H, Schuster FL. Pathogenic and opportunistic free-living amoebae: Acanthamoeba spp., Balamuthia mandrillaris, Naegleria fowleri, and Sappinia diploidea. FEMS Immunol. Med. Microbiol. 2007;50:1-26.

4. Marciano-Cabral F, Cabral G. Acanthamoeba spp. as agents of disease in humans. Clin Microbiol Rev. 2003;16:273-307.

5. Perez-Santonja JJ, Kilvington S, Hughes R, Tufail A, Metheson M, Dart JKG. Persistently culture positive Acanthamoeba keratitis: in vivo resistance and in vitro sensitivity. Ophthalmology. 2003;110:1593-600.

6. Ficker $L$, Seal $D$, Warhurst $D$, Wright $P$. Acanthamoeba keratitis: resistance to medical therapy. Eye. 1990:4:835-8.

7. Aksozek A, McClellan K, Howard K, Niederkorn JY, Alizadeh H. Resistance of Acanthamoeba castellanii cysts to physical, chemical, and radiological conditions. J Parasitol. 2002:88(3):621-3.

8. Lloyd D, Turner NA, Khunkitti W, Hann AC, Furr JR, Russell AD. Encystation in Acanthamoeba castellanii: development of biocide resistance. J Eukaryot Microbiol. 2001;48(1):11-6.

9. Turner NA, Russel AD, Furr JR, Lloyd D. Emergence of resistance to biocides during differentiation of Acanthamoeba castellanii. J Antimicrob Chem. 2000; 46:27-34.

10. Ott I. On the medicinal chemistry of gold complexes as anticancer drugs. Coord Chem Rev. 2009;253:1670-81.

11. Berners-Price SJ, Filipovska A. Gold compounds as therapeutic agents for human diseases. Metallomics. 2011;3:863-73.

12. Yeo Cl, Ooi KK, Akim AM, Ang KP, Fairuz ZA, Halim SNBA, Ng SW, Seng H-L, Tiekink ERT. The influence of $R$ substituents in triphenylphosphinegold(I) carbonimidothioates, $\mathrm{Ph}_{3} \mathrm{PAu}[\mathrm{SC}(\mathrm{OR})=\mathrm{NPh}](\mathrm{R}=\mathrm{Me}$, Et and $\mathrm{PPr})$, upon in vitro cytotoxicity against the HT-29 colon cancer cell line and upon apoptotic pathways. J Inorg Biochem. 2013:127:24-38.

13. Ooi KK, Yeo Cl, Ang K-P, Akim AM, Cheah Y-K, Halim SNA, Seng H-L, Tiekink ERT. Phosphanegold(I) thiolates, $\mathrm{Ph}_{3} \mathrm{PAu}\left[\mathrm{SC}(\mathrm{OR})=\mathrm{NC}_{6} \mathrm{H}_{4} \mathrm{Me}-4\right]$ for $\mathrm{R}=\mathrm{Me}$, Et and $\mathrm{Pr}$, induce apoptosis, cell cycle arrest and inhibit cell invasion of HT-29 colon cancer cells through modulation of the nuclear factor-kB activation pathway and ubiquitination. J Biol Inorg Chem. 2015;20:855-73.

14. Ooi KK, Yeo Cl, Mahandaran T, Ang KP, Akim AM, Cheah Y-K, Seng H-L, Tiekink ERT. G/M cell cycle arrest on HT-29 cancer cells and toxicity assessment of triphenylphosphanegold(I) carbonimidothioates, $\mathrm{Ph}_{3} \mathrm{PAu}[\mathrm{SC}(\mathrm{OR})=\mathrm{NPh}], \mathrm{R}=\mathrm{Me}$, Et, and iPr, during zebrafish development. J Inorg Biochem. 2017;166:173-81.

15. Yeo Cl, Sim JH, Khoo CH, Goh ZJ, Ang KP, Cheah YK, Fairuz ZA, Halim SN, Ng SW, Seng HL, Tiekink ER. Pathogenic Gram-positive bacteria are highly sensitive to triphenylphosphane gold (O-alkylthiocarbamates), $\mathrm{Ph}_{3} \mathrm{PAu}[\mathrm{SC}(\mathrm{OR})=\mathrm{N}(\mathrm{p}$-tolyl)] ( $\mathrm{R}=\mathrm{Me}$, Et and $\mathrm{Pr}$ ). Gold Bull. 2013;46:145-52.

16. Tiekink ERT. Gold derivatives for the treatment of cancer. Crit Rev Hematol Oncol. 2002;42:225-45.

17. Zou T, Lum CT, Lok C-N, Zhang J-J, Che C-M. Chemical biology of anticancer gold(III) and gold(I) complexes. Chem Soc Rev. 2015;44:8786-801.

18. Navarro M. Gold complexes as potential anti-parasitic agents. Coord Chem Rev. 2009:253:1619-26.

19. Fillat MF, Gimeno MC, Laguna A, Latorre E, Ortego L, Villacampa MD. Synthesis, structure and bactericide activity of (Aminophosphane) gold(I) Thiolate complexes. Eur J Inorg Chem. 2011;9:1487-95. 
20. Ray S, Mohan R, Singh JK, Samantaray MK, Shaikh MM, Panda D, Ghosh P. Anticancer and antimicrobial metallopharmaceutical agents based on palladium, gold, and silver N-heterocyclic carbene complexes. J Am Chem Soc. 2007:129:15042-53.

21. Glišic BĐ, Djuran MI. Gold complexes as antimicrobial agents: an overview of different biological activities in relation to the oxidation state of the gold ion and the ligand structure. Dalton Trans. 2014;43:5950-69.

22. Sissons J, Alsam S, Stins M, Ortega-Rivas A, Lorenzo-Morales J, Faull J, Khan NA. Use of In vitro assays to determine effects of human serum in biological characteristics of Acanthamoeba Castellanii. J Clin Microbiol. 2006; 44:2595-600.

23. Sissons J, Kim KS, Stins M, Jayasekera S, Alsam S, Khan NA. Acanthamoeba castellanii induces host cell death via a phosphatidylinositol 3-kinasedependent mechanism. Infect Immun. 2005;73:2704-8.

24. Siddiqui $\mathrm{R}$, Jarroll EL, Khan NA. Balamuthia mandrillaris: role of galactose in encystment and identification of potential inhibitory targets. Exp Parasitol. 2010;126:22-7.

25. Dudley R, Jarroll EL, Khan NA. Carbohydrate analysis of Acanthamoeba castellanii. Exp Parasitol. 2009:122:338-43.

26. Matin A, Stins M, Kim KS, Khan NA. Balamuthia mandrillaris exhibits metalloprotease activities. FEMS Immunol Med Microbiol. 2006:47:83-91.

27. Shaw III CF. Gold-based therapeutic agents. Chem Rev. 1999;99:2589-600.

28. Kean WF, Kean IRL. linical pharmacology of gold. Inflammopharmacology. 2008;16:112-25.

29. Bindoli A, Rigobello MP, Scutari G, Gabbiani C, Casini A, Messori L Thioredoxin reductase: a target for gold compounds acting as potential anticancer drugs. Coord Chem Rev. 2009;253:1692-707.

30. Eisler R. Chrysotherapy: a synoptic review. Inflammation Res. 2003;52: 487-501.

31. Kuntz AN, Davioud-Charvet E, Sayed AA, Califf LL, Dessolin J, Amer ESJ Williams DL. Thioredoxin glutathione reductase from Schistosoma mansoni: an essential parasite enzyme and a key drug target. PLoS Med. 2007;4, e206.

32. Lobanov AV, Gromer S, Salinas G, Gladyshev VN. Selenium metabolism in Trypanosoma: characterization of selenoproteomes and identification of a Kinetoplastida-specific selenoprotein. Nucleic Acids Res. 2006;34:4012-24.

33. Bonilla M, Denicola A, Novoselov SV, Turanov AA, Protasio A, Izmendi D, Gladyshev VN, Salinas G. Platyhelminth mitochondrial and cytosolic redox homeostasis is controlled by a single thioredoxin glutathione reductase and dependent on selenium and glutathione. J Biol Chem. 2008;283:17898-907.

34. Sannella AR, Casini A, Gabbiani C, Messori L, Bilia AR, Vincieri FF, Majori G, Severini C. New uses for old drugs. Auranofin, a clinically established antiarthritic metallodrug, exhibits potent antimalarial effects in vitro: mechanistic and pharmacological implications. FEBS Lett. 2008;582:844-7.

35. Ilari A, Baiocco P, Messori L, Fiorillo A, Boffi A, Gramiccia M, Di Muccio T, Colotti G. A gold-containing drug against parasitic polyamine metabolism: the $X$-ray structure of trypanothione reductase from leishmania infantum in complex with auranofin reveals a dual mechanism of enzyme inhibition. Amino Acids. 2012;42:803-11.

36. Tejman-Yarden N, Miyamoto Y, Leitsch D, Santini J, Debnath A, Gut J, McKerrow JH, Reed SL, Eckman L. A reprofiled drug, auranofin, is effective against metronidazole-resistant Giardia lamblia. Antimicrob Agents Chemother. 2013;57:2029-35

37. Debnath A, Parsonage D, Andrade RM, He C, Cobo ER, Hirata K, Chen S, Garcia-Rivera G, Orozco E, Martinez MB, Gunatilleke SS, Barrios AM, Arkin MR, Poole LB, McKerrow JH, Reed SL. A high-throughput drug screen for Entamoeba histolytica identifies a new lead and target. Nature Med. 2012; 18:956-62.

38. Jackson-Rosario J, Cowart D, Myers A, Tarrien R, Levine RL, Scott RA, Self WT. Auranofin disrupts selenium metabolism in Clostridium difficile by forming a stable Au-Se adduct. J Biol Inorg Chem. 2009;14:507-19.

39. Jackson-Rosario S, Self WT. Inhibition of selenium metabolism in the oral pathogen Treponema denticola. J Bacteriol. 2009;191:4035-40.

40. Novelli F, Recine M, Sparatore F, Juliano C. Gold(I) complexes as antimicrobial agents. Farmaco. 1999;54:232-6.

41. Bertrand B, Casini A. A golden future in medicinal inorganic chemistry: the promise of anticancer gold organometallic compounds. Dalton Trans. 2014:43:4209-19.

42. Dalla Via L, Nardon C, Fregona D. Targeting the ubiquitin - proteasome pathway with inorganic compounds to fight cancer: a challenge for the future. Future Med Chem. 2012:4:525-43.
43. Serratrice M, Edafe F, Mendes F, Scopelliti R, Zakeeruddin SM, Gratzel M, Santos I, Cinellu MA, Casini A. Cytotoxic gold compounds: synthesis, biological characterization and investigation on their inhibition properties of the zinc finger protein PARP-1. Dalton Trans. 2012;41:3287-93.

44. Mendes F, Groessl M, Nazarov AA, Tsybin YO, Sava G, Santos I, Dyson PJ, Casini A. Metal-based inhibition of poly(ADPribose)polymerase the guardian angel of DNA. J Med Chem. 2011;54:2196-206.

45. Schreiber V, Dantzer F, Ame JC, De Murcia G. Poly(ADPribose): novel functions for an old molecule. Nat Rev Mol Cell Biol. 2006;7:517-28.

\section{Submit your next manuscript to BioMed Central and we will help you at every step:}

- We accept pre-submission inquiries

- Our selector tool helps you to find the most relevant journal

- We provide round the clock customer support

- Convenient online submission

- Thorough peer review

- Inclusion in PubMed and all major indexing services

- Maximum visibility for your research

Submit your manuscript at www.biomedcentral.com/submit
) Biomed Central 